\title{
Review of: "Resolution and b value dependent Structural Connectome in ex vivo Mouse Brain"
}

\author{
Francisco Javier Lagos Fritz \\ 1 University Medical Center Hamburg - Eppendorf
}

Potential competing interests: The author(s) declared that no potential competing interests exist.

The paper attempts to extend the optimisation, performance and achievements from the HCP into pre-clinical setups. Therefore, the authors went through the exhaustive process of testing the personally-called "1st diffusion level study". In this level, it is studied how the variation of resolution, b-value and number of b-vectors affects the estimation of microstructure and/or diffusion orientality. From the reported results and discussion, they achieved remarkable results that can be extended upon or, in another context, to help researchers who experiment with small animals like rodents.

However, some problems were found in the submitted pre-print, which I hope it can be corrected before submission into a journal. The most important ones are as follows:

1. Unless it is well known into the pre-clinical community, it is necessary a deeper introduction of why it is relevant to extend the HCP protocol into small animals.

2. Most of the findings are actually expected results when it is compared with human or macaque (e.g. higher resolution, higher definition in the diffusion directions; a b-value of $3000 \mathrm{~s} / \mathrm{mm}^{\wedge} 2$ at 60 directions is the sweet point for HARDI - or at least the minimum required). The missing element then is, if for the authors, their results were expected to be similar to human brain, or if they were expecting something different.

3. The method's section omits relevant information. For example, when the number of b-vectors are subsampled, was that performed on random or in such a way that it is still "evenly" distributed into the diffusion sphere? The different bvalues were achieved at variable diffusion gradient amplitude, duration or variable diffusion time?

4. The DWI that were b-value sub-sampled (e.g. from 8 to 2 shells) were analysed with DTI? Yes, no? If so, I consider that the employed sub-sampling is in itself not recommended, except in the case of $1000-2000 \mathrm{~s} / \mathrm{mm}^{\wedge} 2$. For a higher b-value, it is "advisable" to place an in-between b-value instead (e.g. $2000-4000 \mathrm{~s} / \mathrm{mm}^{\wedge} 2$ ). This is easy to see if it is assumed an "exponential signal decay", where at certain b-value it is not a "significant" difference in the signal attenuation between one an another (e.g. 7000 and $\left.8000 \mathrm{~s} / \mathrm{mm}^{\wedge} 2\right)$

5. It is suggested a writing revision, since the Introduction, Methods and Discussion showed slightly redundancy or issues in the writing coherence. 